\title{
GDPs' perceptions of the WoSCAP oral cancer campaign
}

\author{
General dental practitioners' perceptions of the Awareness Programme oral cancer campaign \\ J. Rodgers and L. M. D. Macpherson BrDent J 2006; 200: 693-697
}

\section{Objectives}

The 2003/04 West of Scotland Cancer Awareness Programme oral cancer campaign was designed to raise public awareness of the signs and symptoms of oral cancer. The objectives of this study were to explore general dental practitioners' (GDPs) awareness and perceptions of the campaign, and its impact on local dental practices.

Methods

A self-completing questionnaire was sent to GDPs in the West of Scotland ( $N=983)$ at the conclusion of the public awareness campaign. Results

A response rate of $68.6 \%$ was achieved. Most dentists (92\%) had heard of the campaign and a large percentage had displayed the promotional materials in their practice. The majority of respondents rated the campaign materials, including a television advertisement, in a very positive manner. Over $40 \%$ of dentists reported that, during the active phase of the campaign, patients had asked for information concerning the programme, and 66\% indicated that registered patients had asked for advice regarding a specific lesion. Additionally, $41 \%$ of dentists reported non-registered patients had attended asking for advice regarding a 'worrying' lesion. Over $60 \%$ of dentists had referred a patient during the campaign and $40 \%$ of these practitioners indicated an increased referral rate during this period.

Conclusions

Most respondents were positive in their assessment of the campaign and reported an increased awareness of oral cancer among patients.

\section{IN BRIEF}

- The oral cancer awareness campaign was received positively by GDPs.

- The campaign's television advertisement had been seen by $78 \%$ of the respondents. The vast majority agreed that it was helpful.

- The campaign appears to have had some impact on practices in the West of Scotland.

- Many dentists reported non-registered patients had attended asking for advice regarding a worrying lesion.

- It will be important to assess the impact of the campaign on patients' awareness of oral cancer over a longer time period.

\section{COMMENT}

The incidence of oral cancer within the UK continues to rise, and has doubled over the last 20 years. There is also a trend towards an increase in younger patients, although the majority are still over the age of 50 . It is noteworthy that of all other possible cancer sites, the West of Scotland Cancer Awareness Programme (WoSCAP) chose oral cancer as their other cancer to 'promote' in 2003, particularly since oral cancer rates in Scotland are twice that of the rest of the UK. The paper by Rodgers and Macpherson explores the impact this promotion had on the general dental practitioners (GDPs) in that region, both with respect to awareness and perception of a disease whose prognosis still remains unacceptably low at $50 \%$.

A self completing questionnaire was sent to all GDPs in the West of Scotland $(n=983)$ at the end of the campaign. A response rate just under $70 \%$ was achieved. Most dentists had heard of the campaign and displayed promotional material. Furthermore $40 \%$ of dentists reported patients asking about the campaign, although the number of such enquiries appeared small. It is perhaps disappointing that although most responding GDPs had received posters, only two thirds displayed them. However, they were very supportive of the advice contained within the TV adverts. Perhaps we should not be so surprised that twice as many GDPs had heard the campaign on the TV as compared to the radio, given the number of broadcasts.

The campaign would appear to have raised the profile of this important condition in the West of Scotland and illustrates the influence of the media. Indeed, stimulating non-registered patients to seek professional advice on a mouth lesion may have other beneficial effects. Encouragingly, GPs were seen to refer patients to their GDP. Campaigns such as this do lead to an increase in referrals that inevitably put a strain on existing clinics and clinical services. Whilst managers will debate the cost utility, a positive diagnosis is priceless to the individual concerned, particularly if caught early.

This campaign adds to the other initiatives in Scotland, such as the Ben Walton Trust and the Scottish Oral Cancer Action Group, that have sought to raise public and professional awareness of oral cancer.

In the meantime, efforts to reduce exposure to the two most important aetiological agents (tobacco and alcohol) combined with a healthy diet of fresh fruit and fresh vegetables, remains the most important message in the prevention of oral cancer.

G. R. Ogden, Professor of Oral \& Maxillofacial Surgery, University of Dundee

DOI: $10.1038 /$ sj.bdj.4813734 